\title{
Consistent Multiparameter Quantisation of GL(n)
}

\author{
A. Sudbery \\ Department of Mathematics \\ University of York \\ Heslington, York, U.K. Y01 5DD \\ and \\ Argonne National Laboratory \\ High Energy Physics Division \\ Argonne, IL 60439
}

April 1990

Abstract

We describe a manifold of quantum group structures on the vector space of the universal enveloping algebra of $\mathrm{gl}(n)$ and on its dual, the space of polynomials in $n^{2}$ variables. The dimension of the manifold is $\left(n^{2}-n+2\right) / 2$.

\section{DISCLAIMER}

This report was prepared as an account of work sponsored by an agency of the United States Government. Neither the United States Government nor any agency thereof, nor any of their employees, makes any warranty, express or implied, or assumes any legal liability or responsibility for the accuracy. completeness, or usefuiness of any information, apparatus, product, or process disclosed, or represents that its use would not infringe privately owned rights. Reference herein to any specific commercial produci, process, or service by trade name, trademark, manufacturer, or otherwise does ro: necessarily constitute or imply its endorsement, recommendation, or favoring by the United States Government or any agency thersoo. The views and opisions of authors expressed herein do not necessarily state or rellect those of the United States Government or any agency thereof.

* Talk given at the Informal Spring Worksiop on Quantum Groups, Argonne National Laboratory, April 26-May 11, 1990. Work supported by the U.S. Department of Energy, Division of High Energy Physics, Contract W-31-109-ENG-38. 


\section{Introduction}

Manin $[1,2]$ has investigated a family of quantum groups containing the algebra of polynomial functions on $G L(n)$ and depending on $N=n(n-1) / 2$ parameters. He found, however, that in this family only the usual one-parameter deformations were consistent with functional independence of the generators in the sense of the Poincare-Birkhoff-Witt theorem, so that the remaining structures were defined on a smaller space than that of the algebra of functions. This paper is devoted to the construction of an $(N+1)$-parameter family of quantum deformations of GL(n) in which the consistency condition is satisfied for all values of the parameters. The algebra is presented both as an algebra generated by $n^{2}$ independent non-commuting matiix elements, with matrix comultiplication, and in the dual form as a deformation $G$ of the universal algebra of $g l(n)$. We start with the latter form, in section 2 ; in section 3 we construct the algebra $G^{*}$ of non-commuting matrix elements by considering the fundamental representation of $G$. In se sion 4 we consider $n=2$ and some other special cases, and comment on the relation of these quantum groups to the Yang-Baxter equation.

\section{Multiparameter Deformation of $\underline{U} \underline{\mathrm{U}(n)}$}

Let $A$ be the polynomial algebra generated by $x_{1}, \ldots x_{n}$ with relations

$$
x_{j} x_{i}=q_{i j} x_{i} x_{j}
$$

where $q_{i j}$ are c-numbers satisfying $q_{i i}=1$ and $q_{j i}=q_{i j}{ }^{-1}$. It will be convenient to define

$$
p_{i j}=\left\{\begin{array}{l}
q_{i j} \text { if } i<j \\
1 \text { if } i \geq j
\end{array}\right.
$$

and to write (1) as

$$
p_{j i} x_{j} x_{i}=p_{i j} x_{i} x_{j}
$$

(no summation convention; throughout this paper all summations will be explicitly marked.)

The algebra $A$ is spanned by the monomials

$$
x^{r}=x_{n}^{r_{n}} \ldots x_{1}^{r_{1}}
$$


with $r=\left(r_{1}, \ldots, r_{n}\right) \in \mathbb{N}^{n}$. We define operators $D_{i}: A \rightarrow A$ and $X_{i j}: A \rightarrow A$ (for $\left.i \neq j\right)$ by

$$
\begin{gathered}
D_{i}\left(x^{r}\right)=r_{i} x^{r} \\
X_{i j}\left(x^{r}\right)=\left[r_{i}\right]_{1 k} x^{r-e_{i}+e_{j}}
\end{gathered}
$$

where $e_{i}$ is the elementary vector whose ith component is $\delta_{i j}$, and

$$
[x]_{u}=\frac{u^{x}-u^{-x}}{u-u^{-1}}
$$

$u$ being a further independent parameter. These operators satisfy

and

$$
D_{i}\left(x^{r} x^{s}\right)=\left(D_{i} x^{r}\right) x^{s}+x^{r}\left(D_{i} x^{s}\right)
$$

where

$$
X_{i j}\left(x^{r} z^{s}\right)=\left(X_{i j} x^{r}\right) \frac{u^{s_{i}} a_{j} s}{a_{i}{ }^{s}} x^{s}+\frac{u^{-r_{i}} b_{j}^{r}}{b_{i}{ }^{r}} x^{r}\left(X_{i j} x^{s}\right)
$$

$$
a_{i}{ }^{s}=p_{i 1}^{s_{1}} \ldots p_{i n}^{s_{n}}, \quad b_{i}^{r}=p_{1 i}^{r_{1}} \ldots p_{n i}^{{ }^{r}}
$$

Thus $D_{i}$ and $X_{i j}$ are generalised (twisted) derivations of $A$ with the coproducts

$$
\Delta\left(D_{i}\right)=D_{i} \otimes 1+1 \otimes D_{i}
$$

and

$$
\Delta\left(X_{i j}\right)=X_{i j} \otimes u^{D_{i}} A_{i}^{-1} A_{j}+u^{-D_{i}} B_{i}^{-1} B_{j} \otimes X_{i j}
$$

where $A_{i}$ and $B_{i}$ are the operators (functions of $D_{1}, \ldots D_{n}$ ) whose eigenvalues are given by (8).

Eq. (7) remains true if $u$ is replaced by $u^{-1}$; thus we could also choose the coproduct obtained 
from (10) by changing $u$ to $u^{-1}$.

Let

$$
E_{i}=X_{i, i+1} ; \quad F_{i}=X_{i+1, i}
$$

then $D_{i}, E_{i}$ and $F_{i}$ satisfy

$$
\begin{gathered}
{\left[D_{i}, D_{j}\right]=0} \\
{\left[D_{i}, E_{j}\right]=\left(-\delta_{i j}+\delta_{i, j+1}\right) E_{j},} \\
{\left[D_{i}, F_{j}\right]=\left(\delta_{i j}-\delta_{i, j+1}\right) F_{j},} \\
{\left[E_{i}^{r}, F_{j}\right]=\left[D_{i+1}-D_{i}\right]_{u} ;} \\
E_{i \pm 1} E_{i}^{2}+E_{i}^{2} E_{i \pm 1}=\left(u+u^{-1}\right) E_{i} E_{i \pm 1} E_{i} \\
F_{i \pm 1} F_{i}^{2}+F_{i}^{2} F_{i \pm 1}=\left(u+u^{-1}\right) F_{i} F_{i \pm 1} F_{i}
\end{gathered}
$$

and

$$
\left[E_{j}, E_{j}\right]=\left[F_{i}, F_{j}\right]=0 \quad \text { if } \quad|i-j| \geq 2
$$

In view of (9) and (10) and the remark following them, we choose coproducts

$$
\begin{gathered}
\Delta\left(D_{i}\right)=D_{i} \otimes 1+1 \otimes D_{i} \\
\Delta\left(E_{i}\right)=E_{i} \otimes u^{D_{i}} R_{i}+u^{-D_{i}} C_{i} \otimes E_{i} \\
\Delta\left(F_{i}\right)=F_{i} \otimes u^{-D_{i+1}} R_{i}^{-1}+u^{D_{i+1}} C_{i}^{-1} \otimes F_{i}
\end{gathered}
$$

where

$$
\begin{aligned}
& R_{i}=A_{i}^{-1} A_{i+1}=\prod_{k=1}^{n}\left[\frac{p_{i+1, k}}{P_{i k}}\right]^{D_{k}}, \\
& C_{i}=B_{i}^{-1} B_{i+1}=\prod_{k=1}^{n}\left[\frac{p_{k, i+1}}{p_{k i}}\right]^{D_{k}} .
\end{aligned}
$$

The commutators (11) give rise to the following relations between $D_{i}, E_{i}, F_{i}, R_{i}$ and $C_{i}$ :

$$
\left.\begin{array}{ll}
E_{i} f\left(D_{i}\right)=f\left(D_{i}+1\right) E_{i}, & E_{i} f\left(D_{i+1}\right)=f\left(D_{i+1}-1\right) E_{i} \\
F_{i} f\left(D_{i}\right)=f\left(D_{i}-1\right) F_{i}, & F_{i} f\left(D_{i+1}\right)=f\left(D_{i+1}+1\right) F_{i}
\end{array}\right\}
$$


for any function $f$; and

$$
\left.\begin{array}{ll}
C_{j} E_{i}=s_{i j} E_{i} C_{j}, & C_{j} F_{i}=s_{i j}^{-1} F_{i} C_{j} \\
R_{j} E_{i}=s_{j i} E_{i} R_{j}, & R_{j} F_{i}=s_{j i}^{-1} F_{i} R_{j}
\end{array}\right\}
$$

where

$$
s_{i j}=\frac{p_{i j} p_{i+1, j+1}}{p_{i, j+1} p_{i+1, j}}
$$

Using these, it is straightforward to verify that the algebra $G$ generated by $D_{i}, E_{i}$ and $F_{i}$, with relations (11-13) and comultiplication (14), is a bialgebra $[2,3,4]$, i.e. that the coproducts (14) are compatible with the relations (11-13). It becomes a Hopf algebra when furnished with the ustal counit and the antipode

$$
\begin{gathered}
S\left(D_{i}\right)=-D_{i}, \\
S\left(E_{i}\right)=-u^{-1} R_{i}^{-1} E_{i} C_{i}^{-1}, \\
S\left(F_{i}\right)=-u^{-1} R_{i} F_{i} C_{i} .
\end{gathered}
$$

\section{The Dual Form}

Let $V^{*}$ be the $n$-dimensional vectcr spece spanned by $x_{1}, \ldots, x_{n}$ and $V$ its ulual. We think of the elements of $V^{*}$ as coordinate functions on $V$, so that the algebra $A$ generated by $V^{*}$ is a coordinate algebra on $V$ [5]. Eqs. (3) and (4), restricted to $V^{*}$, define a representation $\rho: G \otimes V^{*} \rightarrow V^{*}$ of our quantum group $G$ in which, with respect to the basis $x_{1}, \ldots, x_{n}$, the generators $D_{i}, E_{i}$ and $F_{i}$ are represented by elementary diagonal, subdiagonal and superdiagonal matrices respectively. We denote by $a_{i j}$ the matrix elements of this representation, regarded as functions on $G$, i.e. elements of the dual $G^{*} ;$ then

$$
\left.\begin{array}{c}
\left\langle a_{i j}, D_{k}\right\rangle=\delta_{i k} \delta_{j k} \\
\left\langle a_{i j}, E_{k}\right\rangle=\delta_{i, k+1} \delta_{j k} \\
\left\langle a_{i j}, F_{k}\right\rangle=\delta_{i k} \delta_{j, k+1}
\end{array}\right\}
$$

where the angle brackets denote the pairing between $G$ and $G^{*}$. 
The transposes of these matrices yield $\rho^{*}: G \otimes V \rightarrow V$ which is an antirepresentation of $G$, i.e. a representation of the oppositc algebra $G^{\mathrm{op}}$. Dualising this gives a map $\delta: V^{*} \rightarrow G^{*} \otimes V^{*}$ given by

$$
\delta\left(x_{i}\right)=\sum_{k} a_{k i} \otimes x_{k} \quad \text { or, in matrix notation, } \quad \delta\left(x^{\top}\right)=x^{\top} \otimes A .
$$

The statement that each $\rho(g)$ is a generalised derivation of $A$ with the comultiplication $\Delta$ dualises to the statement that $\delta$ extends to a homomorphism $\delta: A \rightarrow G^{*} \otimes A$ (in the terminology of [5], the coordinate algebra $A$ is compatible with the representation $p^{*}$ ). Hence (2) is satisfied by $\sum_{k} a_{k i} \otimes x_{k}$, and so

$$
\sum_{k l} \frac{p_{i j}}{p_{k l}} a_{k i} a_{l j} \otimes y_{k l}=\sum_{k l} \frac{p_{j i}}{p_{l k}} a_{l j} a_{k i} \otimes y_{l k}
$$

where $y_{k l}=p_{k l} x_{k} x_{l}$. Since these satisfy $y_{k l}=y_{l k}$ (see (2)) but are otherwise independent, it follows that

$$
\frac{p_{i j}}{p_{k i}} a_{k i} a_{l j}+\frac{p_{i j}}{p_{l k}} a_{l i} a_{k j}=\frac{p_{j i}}{p_{k l}} a_{k j} a_{l i}+\frac{p_{j i}}{p_{k l}} a_{k j} a_{l i}
$$

These relations are compatible with matrix comultiplication

$$
\Delta\left(a_{i j}\right)=\sum_{k} a_{i k} \otimes a_{k j}
$$

(i.e. $\Delta\left(a_{i j}\right)$ satisfy (19) if $a_{i j}$ do) since this is the comultiplication in $G^{*}, a_{i j}$ being matrix elements of a representation of $G$.

Eq. (19) constitutes half of the relations obtained by Manin [1] for the matrices of operators on a quantum superspace in which all the coordinates are even. In order to generate a deformation of the algebra of polynomials in $n^{2}$ commuting variables, the $a_{i j}$ mist satisfy an equal number of further independent relations. Instead of the remaining relations postulated by Manin, our $a_{i j} \in G^{*}$ satisfy

$$
\frac{p_{i j}^{\prime}}{p_{k l}^{\prime}} a_{i k} a_{j l}+\frac{p_{i j}^{\prime}}{p_{J k}^{\prime}} a_{i j} a_{j k}=\frac{p_{j i}^{\prime}}{p_{k l}^{\prime}} a_{j k} a_{i l}+\frac{p_{j i}^{\prime}}{p_{k l}^{\prime}} a_{j k} a_{i l}
$$


where

$$
p^{\prime}= \begin{cases}u^{2} q_{i j}{ }^{-1} & \text { if } i<j \\ 1 & \text { if } i \geq j\end{cases}
$$

These relations could not have been anticipated from the construction of the $a_{i j}$. To prove them, note that they are the conditions on the matrix $A=\left(a_{i j}\right)$ for the map $x \rightarrow A \otimes x$ to preserve the relations $p^{\prime}{ }_{j i} x_{j} x_{i}=p^{\prime}{ }_{i j} x_{i} x_{j}$, and therefore they are compatible with the matrix corsultiplication (20). It follows from this that to prove that they hold in $G^{*}$ it is sufficient to verify them when bracketed with the generators $D_{i}, E_{i}$ and $F_{i}$ of $G$. This is readily done, using the brackets (18) and the coproducts (14) together with the definition

$$
\left\langle a_{i j} a_{k l}, X\right\rangle=\left\langle a_{i j} \otimes a_{k l}, \Delta(X)\right\rangle \text { for } X \in G .
$$

The relations (19) and (21) can also be related to algebras of q-anticommuting coordinates which are dual to the q-commuting coordinates considered above. The full set of conditions is as follows:

$$
\begin{aligned}
& \Leftrightarrow \quad x^{\top} \rightarrow x^{\top} A \text { preserves } p_{j i} x_{j} x_{i}=p_{i j} x_{i} x_{j} \\
& \Leftrightarrow \quad \xi \rightarrow A \xi \text { preserves } p_{i j} \xi_{j} \xi_{i}=-p_{j i} \xi_{i} \xi_{j} \text { and } \xi_{i}{ }^{2}=0 ; \\
& \Leftrightarrow \quad x \rightarrow A x \text { preserves } p_{j i}^{\prime} x_{j} x_{i}=p_{i j}^{\prime} x_{i} x_{j} \\
& \Leftrightarrow \quad \xi^{\top} \rightarrow \xi^{\top} A \text { preserves } p_{i j}^{\prime} \xi_{j} \xi_{i}=-p^{\prime}{ }_{j i} \xi_{i} \xi_{j} \text { and } \xi_{i}{ }^{2}=0 .
\end{aligned}
$$

The difference between this algebra and that of ref. [1] is that in the latter $p_{i j}{ }_{i j}$ is taken to be equal to $p_{i j}$. However, it can be shown that they must be related by (22), for some value of $u$ : for the algebra defined by (19) and (21) to have the following consistency property.

The relations (19) and (21) refer to a $2 \times 2$ submatrix of $A$. Suppose $i<j$ and $k<l$, and write

$$
\begin{gathered}
{\left[\begin{array}{ll}
a & b \\
c & d
\end{array}\right]=\left[\begin{array}{ll}
a_{i k} & a_{i l} \\
a_{j k} & a_{j l}
\end{array}\right],} \\
q=q_{i j}, \quad p=u^{2} q_{k j}^{-1} .
\end{gathered}
$$


Then (19) and (21) can be written as

$$
\begin{gathered}
b a=p a b, \quad d b=q b d, \\
c a=q d c, \quad d c=p c d, \\
c b=q p^{-1} b c, \\
d a=u^{-2} p q a d+\left(1-u^{-2}\right) q b c .
\end{gathered}
$$

These relations enable any monomial in the $a_{i j}$ to be expressed as a sum of lexicographically ordered monomials. The reordering procedure is not unique, but Manin [1] has showin that the different possible procedures will lead to the same result for all monomizls if they do so for cubic monomials, when there are just two possible ways of reordering $z y x$ to $x y z$

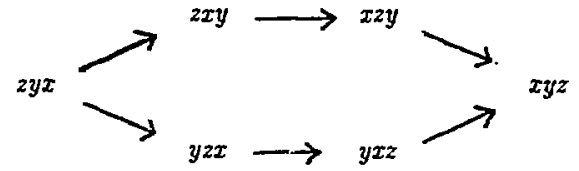

By considering all possible relative positions of the three elements $x, y, z$ in the matrix $A$, it can be verified that the two ways of reordering $z y x$, using (24), give the same result in all cases. It follows that the lexicographically oredered monomials are independent and form a basis of $G^{*}$.

\section{3. $n=2$ and Other Special Cases}

When $n=2$ the three parameters $p, q, u$ are related by $u^{2}=p q$ and the last relation of (24) becomes

$$
d a-a d=q b c-q^{-1} c b .
$$

The relations (24) can now be expressed in the form

$$
\sum_{k l} R_{i j, k j} a_{k m} a_{l n}=\sum_{k l} a_{j l} a_{i k} R_{k l, m n}
$$

with the $R$-matrix 


$$
R=\left[\begin{array}{cccc}
p q & 0 & 0 & 0 \\
0 & q & p q-1 & 0 \\
0 & 0 & p & 0 \\
0 & 0 & 0 & p q
\end{array}\right]
$$

(with rows and columns labelled by $i j$ and $k l$ in the order $11,12,21,22$ ). This satisfies the Yang-Baxter equation.

If $q=p$ this gives the familiar one-parameter deformation $[2,3]$ of $g l(2)$. If $q=p^{-1}$ the $R$-matrix is in factorised form

$$
R=Q \otimes Q^{-1} \quad \text { where } \quad Q=\left[\begin{array}{ll}
1 & 0 \\
0 & q
\end{array}\right]
$$

and the relations in the algebra $G^{*}$ can be written as

$$
a_{i k l} a_{i j}=q^{i-j-k+d} a_{i j} a_{k l} .
$$

For all values of $p$ and $q$ the algebra defined by (24) and (26) has the power property noted by Corrigan et al $[6,7]$ : if the matrix elements of $A$ satisfy these relations, then those of $A^{n}$ satisfy similar reletions with $(q, p)$ replaced by $\left(q^{n}, p^{n}\right)$.

For general $n$, the usual one-parameter deformation $[2,3]$ of $g l(n)$ is obtained by taking all the $q_{i j}$ equal to $q$ for $i<j$, and $u=q$. The resulting algebra is quasitriangular, i.e. is described by an $R$-matrix which satisfies the Yang-Baxter equation. This is also true for general $q_{i j}$ if $u=1$, when the relations (19) and (21) become

$$
q_{i j} a_{i k} a_{j l}=q_{k l} a_{j l} a_{i k},
$$

which is the same as (27) with the $R$-matrix

$$
R_{i j, k i}=q_{i j} \delta_{i k} \delta_{j l}
$$

If $q_{i j}$ factorises as $q_{i j}=q_{i} q_{j}^{-1}$ to give an $R$-matrix $R=Q \otimes Q^{-1}$, then the $n \times n$ matrix $A$ has the power property that the elements of $A^{n}$ satisfy (29) with $q_{i j}$ replaced by $q_{i j}{ }^{n}$. 


\section{References}

1. Yu.I.Manin, Multiparametric Quantum Deformation of the General Linear Supergroup.

Commun. Math. Phys. 123 (1989), 163-175.

2. Yu. I. Manin, Quantum Groups and Non-commutative Geometry. CRM, Montreal 1988.

3. V.G.Drinfel'd, Quantum Groups. Proc. I.C.M. Berkeley (AMS, 1987), 798-820.

4. S. Maịid, Quasitriangular Hopf Algebras and Yang-Baxter Equations.

Int. J. Mod. Phys. A 5, 1-91 (1990)

5. A. Sudbery, Coordinate Algebras for Representation Spaces of Quanturn Groups.

York preprint, 1989.

6. E.Corrigan, D.B.Fairlie and P.Fletcher, Some Aspects of Quantum Groups and Supergroups.

J. Math. Phys. (to be published).

7. S. Vokos, J. Wess and B. Zumino, Properties of Quantum $2 \times 2$ Matrices.

LAPP preprint, 1989. 